\title{
A High-Resolution Technique for Multidimensional NMR Spectroscopy
}

\author{
Ye (Geoffrey) Li, Javad Razavilar, and K. J. Ray Liu,* Senior Member, IEEE
}

\begin{abstract}
In this paper, a scheme for estimating frequencies and damping factors of multidimensional nuclear magnetic resonance (NMR) data is presented. multidimensional NMR data can be modeled as the sum of several multidimensional damped sinusoids. The estimated frequencies and damping factors of multidimensional NMR data play important roles in determining protein structures. In this paper we present a high-resolution subspace method for estimating the parameters of NMR data. Unlike other methods, this algorithm makes full use of the rank-deficiency and Hankel properties of the prediction matrix composed of NMR data. Hence, it can estimate the signal parameters under low signal-to-noise ratio (SNR) by using a few data points. The effectiveness of the new algorithm is confirmed by computer simulations and it is tested by experimental data.
\end{abstract}

Index Terms-Damped sinusoids, high resolution, multidimensional NMR, parameter estimation.

\section{INTRODUCTION}

$\mathbf{M}$ ULTIDIMENSIONAL nuclear magnetic resonance (NMR) data can be modeled as the sum of multidimensional damped sinusoids. The frequencies and damping factors of damped sinusoids are crucial to determining protein structures using NMR spectroscopy. The frequency resolution of the fast Fourier transform (FFT)-based algorithms [1], [2] is limited by the short acquisition time of the NMR data and measurement noise. Hence, to improve the resolution, many model-based methods [3]-[27] have been proposed for the parameter estimation of one-dimensional (1-D) and twodimensional (2-D) NMR data. The autoregressive modeling of NMR data is one of the most commonly used algorithms in the analysis of 1-D NMR data [8]. In particular, it has been shown that the methods based on the linear prediction technique can estimate parameters more accurately than the standard FFT methods do. This should also be true for 2-D NMR data.

Unfortunately, the existing model-based parameter estimation algorithms for 2-D NMR data are still sensitive to measurement noise, which limits their frequency resolution. The

Manuscript received January 24, 1997; revised July 24, 1997. This work was supported in part by the National Institutes of Health (NIH) under Grant 1R01GM49707 and by the National Science Foundation (NSF) under Grant MIP9457397. This work was done when Y. Li was at the University of Maryland at College Park. Asterisk indicates corresponding author.

Y. Li is with AT\&T Labs-Research, Red Bank, NJ 07701-7033 USA

J. Razavilar is with the Electrical Engineering Department and Institute for Systems Research, University of Maryland at College Park, MD 20742 USA.

*K. J. Ray Liu is with the Electrical Engineering Department and Institute for Systems Research, University of Maryland at College Park, MD 20742 USA (e-mail: kjrliu@isr.umd.edu).

Publisher Item Identifier S 0018-9294(98)00247-X. subspace methods [19], [20], which provide high-resolution estimation in many signal processing applications, are good candidates for NMR spectroscopy to further improve the frequency resolution. The Multiple signal classification (MUSIC) algorithm [9] is one of the most effective and commonly used algorithms for 1-D stationary signals. The MUSIC algorithm can even achieve the Cramér-Rao lower bound under some mild conditions. However, the NMR signals consist of damped sinusoids, which is, therefore, nonstationary. Therefore, the original MUSIC algorithm cannot be directly applied to NMR data. In this paper, we present a novel parameter estimation method for NMR data based on the subspace techniques, which we will call M-D DMUSIC algorithm in order to reflect its capability to estimate the parameters (frequencies and damping factors) of multidimensional damped sinusoids. Since M-D MUSIC algorithm makes full use of the rank-deficiency and Hankel properties of the prediction matrix composed of NMR data, it can estimate the signal parameters under low signal-tonoise ratios (SNR's) by using only few data. The effectiveness of the new algorithm is demonstrated by computer examples and the experimental data obtained from National Institutes of Health $(\mathrm{NIH})$.

The rest of the paper is organized as follows. After a brief description of the mathematical model of NMR signals in Section II, a damped MUSIC (DMUSIC) algorithm for estimating the parameters of 1-D NMR signals will be presented and analyzed in Section III. In Section IV, the DMUSIC algorithm will be generalized to multidimensional NMR signals, by first extending the results to 2-D NMR signals and then to general case of M-D NMR signals. A low-complexity peak-searching algorithm for searching the peaks of M-D DMUSIC spectrum is described in Section V. Computer examples are presented in Section VI and the application of the 2-D MUSIC algorithm to a set of experimental data from NIH is described in Section VII.

\section{MATHEMATiCAL Model of NMR Signals}

Before developing the DMUSIC algorithm for multidimensional NMR data, we briefly describe the mathematical model of M-D NMR data here. For a comprehensive review of NMR spectroscopy, interested readers should refer to [23]-[25].

Since the multidimensional NMR signal is the extension of the 2-D NMR signal, we first introduce the mathematical model of 2-D NMR signals. A 2-D NMR signal can be 
expressed by a continuous hypercomplex form $X_{h}\left(t_{1}, t_{2}\right)$ as [23]-[25]

$$
\begin{aligned}
& X_{h}\left(t_{1}, t_{2}\right)= \\
& \sum_{k=1}^{K} a_{k}\left\{\cos \left(\Omega_{k}^{(1)} t_{1}+\theta_{k}^{(1)}\right)\right. \\
& \quad \cdot \cos \left(\Omega_{k}^{(2)} t_{2}+\theta_{k}^{(2)}\right) \\
& \quad+i \sin \left(\Omega_{k}^{(1)} t_{1}+\theta_{k}^{(1)}\right) \cos \left(\Omega_{k}^{(2)} t_{2}+\theta_{k}^{(2)}\right) \\
& \quad+j \cos \left(\Omega_{k}^{(1)} t_{1}+\theta_{k}^{(1)}\right) \sin \left(\Omega_{k}^{(2)} t_{2}+\theta_{k}^{(2)}\right) \\
& \left.\quad+i j \sin \left(\Omega_{k}^{(1)} t_{1}+\theta_{k}^{(1)}\right) \sin \left(\Omega_{k}^{(2)} t_{2}+\theta_{k}^{(2)}\right)\right\} \\
& \cdot e^{-t_{1} / T_{k}^{(1)}-t_{2} / T_{k}^{(2)}}
\end{aligned}
$$

where $K$ is the model order, $\Omega_{k}^{(1)}$ and $\Omega_{k}^{(2)}$ denote the angular frequencies of the magnetization corresponding to $t_{1}$ and $t_{2}$, respectively, $T_{k}^{(1)}$ and $T_{k}^{(2)}$ are the decay constants of the magnetization, and $a_{k}$ 's are the amplitudes of damped sinusoids.

In our discussion, we employ the commonly used complex representation $X_{c}\left(t_{1}, t_{2}\right)$, that can be obtained by letting $i=j$ and $i j=-1$ in [2]

$$
X_{c}\left(t_{1}, t_{2}\right)=\sum_{k=1}^{K} c_{k} e^{\left(-\gamma_{k}^{(1)}+j \Omega_{k}^{(1)}\right) t_{1}+\left(-\gamma_{k}^{(2)}+j \Omega_{k}^{(2)}\right) t_{2}}
$$

where $c_{k}=a_{k} e^{j\left(\theta_{k}^{(1)}+\theta_{k}^{(2)}\right)}$, and $\gamma_{k}^{(1)}=1 / T_{k}^{(1)}, \gamma_{k}^{(2)}=1 / T_{k}^{(2)}$ which are called the decay rates.

If the continuous complex 2-D NMR signal is measured at uniform intervals, $\Delta_{1}$ for $t_{1}$ and $\Delta_{2}$ for $t_{2}$, a set of 2-D NMR data $\left\{x\left(n_{1}, n_{2}\right)\right\}$ will be obtained as

$$
x\left(n_{1}, n_{2}\right)=\sum_{k=1}^{K} c_{k} e^{s_{k}^{(1)} n_{1}+s_{k}^{(2)} n_{2}}
$$

where $s_{k}^{(l)}=-\alpha_{k}^{(l)}+j \omega_{k}^{(l)}$, and $\omega_{k}^{(l)}=\Omega_{k}^{(l)} \Delta_{l}, \alpha_{k}^{(l)}=\gamma_{k}^{(l)} \Delta_{l}$ for $l=1,2$ with $\alpha_{k}^{(l)}$ being the damping factor. Without loss of generality, we assume that $\boldsymbol{s}_{k}=\left(s_{k}^{(1)}, s_{k}^{(2)}\right)$ for $k=$ $1,2, \cdots, K$ are distinct. It should be pointed out that high SNR is required in order to estimate the signals with only distinct $\alpha_{k}$ 's, but the same $\omega_{k}$ 's, as demonstrated by Fig. 1(d)-(e). In presence of measurement error or noise $w\left(n_{1}, n_{2}\right)$, the measured NMR data can be expressed as

$$
y\left(n_{1}, n_{2}\right)=x\left(n_{1}, n_{2}\right)+w\left(n_{1}, n_{2}\right)
$$

for $n_{i}=0,1, \cdots, N_{i}-1$ and $i=1,2$. In the above expression $N_{i}$ 's are the acquisition times of each time domain. We will assume $N_{1}=N_{2}=N$ in our discussion.

In (3) and (4), if we let $N_{2}=1$, then the mathematical model of 2-D NMR signal is degenerated into that of 1-D NMR signal, which can be rewritten as

$$
y(n)=x(n)+w(n)
$$

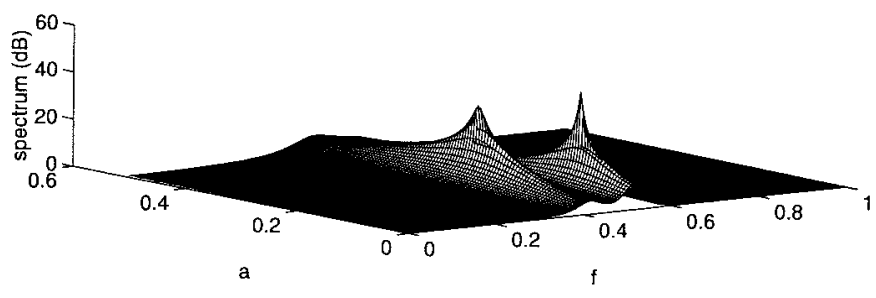

(a)

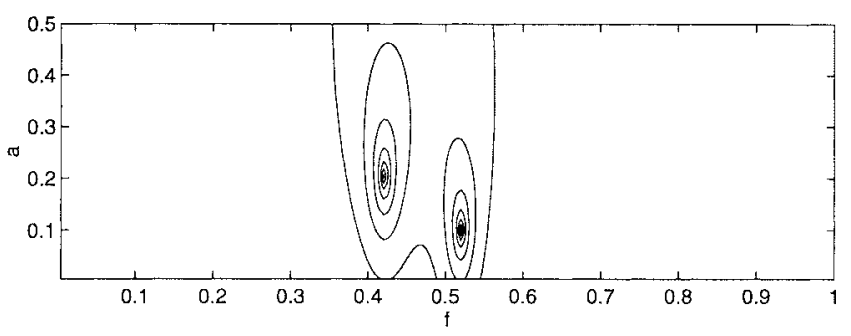

(b)

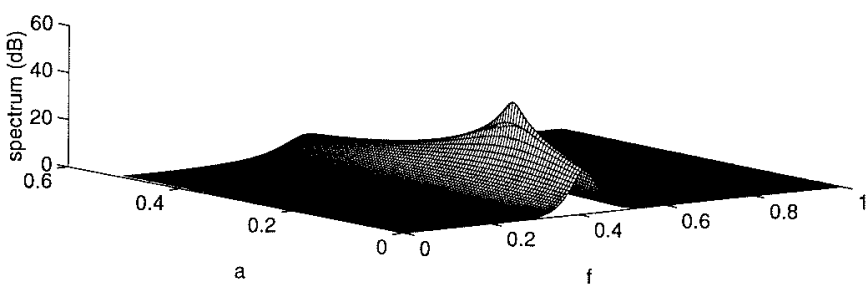

(c)

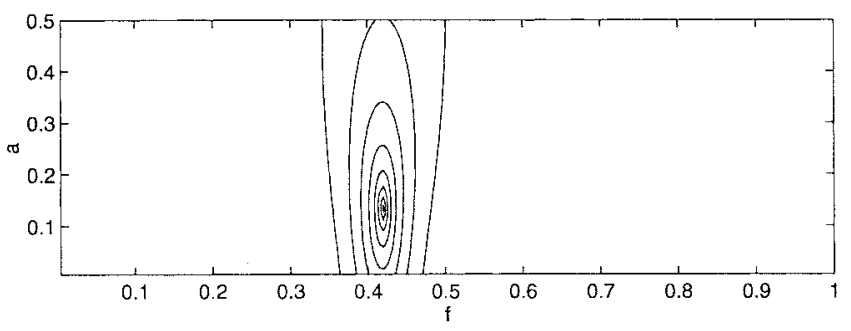

(d)

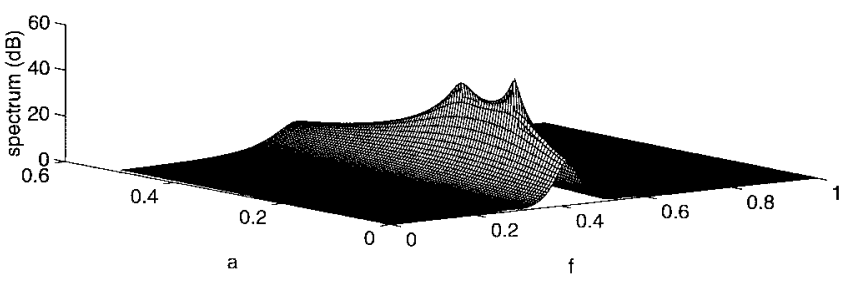

(e)

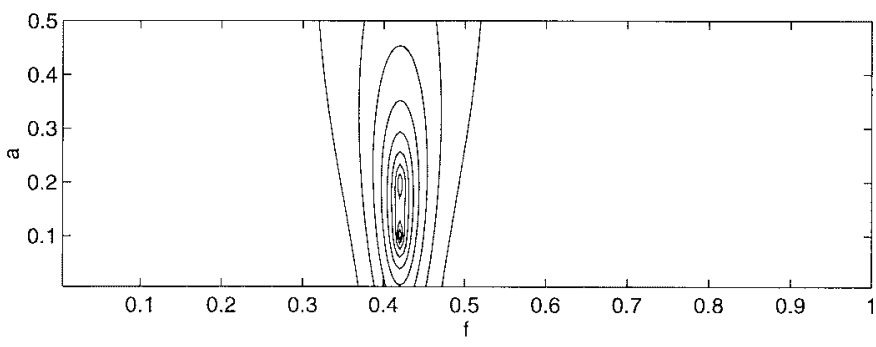

(f)

Fig. 1. Spectrum and contour of DMUSIC algorithm: (a) and (b) when $s_{1}=-0.2+j 2 \pi 0.42, s_{2}=-0.1+j 2 \pi 0.52$ and SNR $=40 \mathrm{~dB} ;$ (c) and (d) when $s_{1}=-0.2+j 2 \pi 0.42, s_{2}=-0.1+j 2 \pi 0.42$ and SNR $=$ $40 \mathrm{~dB}$; (e) and (f) when $s_{1}=-0.2+j 2 \pi 0.42, s_{2}=-0.1+j 2 \pi 0.42$ and $\mathrm{SNR}=60 \mathrm{~dB}$. 
where $w(n)$ denotes again the measurement noise and

$$
x(n)=\sum_{k=1}^{K} c_{k} e^{s_{k} n}
$$

for $n=0,1, \cdots, N-1$. The mathematical model of 2-D NMR signal can be easily extended to that of $L$-dimensional NMR signal as

$$
y(\boldsymbol{n})=x(\boldsymbol{n})+w(\boldsymbol{n})
$$

and

$$
x(\boldsymbol{n})=\sum_{k=1}^{K} c_{k} e^{\boldsymbol{s}_{k} \boldsymbol{n}^{T}}
$$

for $\boldsymbol{n} \in\{0,1, \cdots, N-1\}^{L}$. In the above expression, $\boldsymbol{n}=$ $\left[n_{1}, \cdots, n_{L}\right]$ is a time-index vector and $\boldsymbol{s}_{k}=\left[s_{k}^{(1)}, \cdots, s_{k}^{(L)}\right]$ is a complex frequency vector. Similar to the 2 -D case, $w(\cdot)$ represents measurement noise and $K$ denotes the model order. Normally, we have to make sure $N>2 K$, to be able to estimate signal parameters.

\section{1-D DMUSIC ALGORITHM}

We first present the DMUSIC algorithm for 1-D NMR signals (1-D MUSIC algorithm). To derive 1-D DMUSIC algorithm, we will set up an $(N-J) \times J$ prediction matrix

$$
A=\left(\begin{array}{cccc}
y(0) & y(1) & \cdots & y(J-1) \\
y(1) & y(2) & \cdots & y(J) \\
\vdots & \vdots & \vdots & \vdots \\
y(N-J-1) & y(N-J) & \cdots & y(N-1)
\end{array}\right)
$$

where $K \leq J \leq N-K$. We normally choose $J=\lceil N / 2\rceil$ to obtain the best performance [4]. The prediction matrix in the DMUSIC algorithm plays a similar role as the correlation matrix in the MUSIC algorithm [20]. From (5) and (6), $A$ can be written as (see also, [27])

$$
\boldsymbol{A}=\sum_{k=1}^{K} c_{k} \boldsymbol{r}_{l}\left(s_{k}\right) \boldsymbol{r}_{r}^{T}\left(s_{k}\right)+\boldsymbol{W}=\boldsymbol{S}_{l} C \boldsymbol{S}_{r}^{T}+\boldsymbol{W}
$$

where $\boldsymbol{r}_{r}(s)$ and $S_{r}$ are the right signal vector and the right signal matrix, respectively, defined as

$$
\begin{aligned}
\boldsymbol{r}_{r}\left(s_{k}\right) & =\left(\begin{array}{c}
1 \\
e^{s_{k}} \\
\vdots \\
e^{(J-1) s_{k}}
\end{array}\right) \\
\boldsymbol{S}_{r} & =\left[\boldsymbol{r}_{r}\left(s_{1}\right), \boldsymbol{r}_{r}\left(s_{2}\right), \cdots, \boldsymbol{r}_{r}\left(s_{K}\right)\right]
\end{aligned}
$$

respectively. The left signal vector $\boldsymbol{r}_{l}(s)$ and the left signal matrix $\boldsymbol{S}_{\boldsymbol{l}}$ are similarly defined. $\boldsymbol{C}$ is a $K \times K$ diagonal matrix with $\operatorname{diag}(C)=\left(c_{1}, c_{2}, \cdots, c_{K}\right)$. The noise matrix $W$ is given by

$$
W=\left(\begin{array}{cccc}
w(0) & w(1) & \cdots & w(J-1) \\
w(1) & w(2) & \cdots & w(J) \\
\vdots & \vdots & \vdots & \vdots \\
w(N-J-1) & w(N-J) & \cdots & w(N-1)
\end{array}\right) .
$$

If $s_{k}$ 's are distinct, then $\boldsymbol{r}_{r}\left(s_{k}\right)$ for $k=1,2, \cdots, K$ are linearly independent, hence $S_{r}$ is full column rank, and so is $S_{l}$. Since the rank of $\boldsymbol{C}$ is $K$, the rank of $A$ is also equal to $K$ when there is no noise. Since there are many effective algorithms to estimate the model order $K$ [28], [29], in our algorithm we will assume that $K$ is known in advance. In [27], the authors have used a similar approach to derive their state space formulation. If there is no noise, by means of singular value decomposition, $A$ can be decomposed into the product of three matrices

$$
\boldsymbol{A}=\boldsymbol{U} \boldsymbol{D} \boldsymbol{V}^{H}
$$

where $\boldsymbol{U}$ and $V$ are unitary matrices, $D$ is a diagonal matrix, and $H$ denotes Hermitian, i.e., conjugate transpose, operator. Furthermore, $\boldsymbol{D}$ must be of the form

$$
\begin{array}{r}
\operatorname{diag}(\boldsymbol{D})=\left(\sigma_{1}, \sigma_{2}, \cdots \sigma_{K}, 0, \cdots, 0\right), \\
\sigma_{1} \geq \sigma_{2} \geq \cdots \geq \sigma_{K} .
\end{array}
$$

From (13), we also have

$$
A V=U D .
$$

Denote $\boldsymbol{v}_{i}$ the $i$-th column of $\boldsymbol{V}, \operatorname{span}\left\{\boldsymbol{v}_{1}, \cdots, \boldsymbol{v}_{K}\right\}$ is called the signal subspace since

$$
\operatorname{span}\left\{\boldsymbol{v}_{1}, \cdots, \boldsymbol{v}_{K}\right\}=\operatorname{span}\left\{\boldsymbol{r}_{r}\left(s_{1}\right), \cdots, \boldsymbol{r}_{r}\left(s_{K}\right)\right\}
$$

where $\operatorname{span}\{\}$ is referred to as the subspace that is defined by the set of all linear combinations of the vectors. From (14) and (15), we have the following orthogonality relations

$$
A V_{n}=\mathbf{0}, \quad \text { or } \quad \boldsymbol{A} \boldsymbol{v}_{k}=\mathbf{0} \text { for } k=K+1, \cdots, J
$$

where $\boldsymbol{V}_{\boldsymbol{n}}=\left[\boldsymbol{v}_{K+1}, \cdots, \boldsymbol{v}_{L}\right]$. From (10), we have

$$
S_{l} C S_{r}^{T} v_{k}=\mathbf{0} \text { for } k=K+1, \cdots, J .
$$

Since both $S_{l}, S_{r}$ and $\boldsymbol{C}$ are full rank, $\boldsymbol{S}_{r}^{T} \boldsymbol{v}_{\boldsymbol{k}}=\mathbf{0}$ for $k=K+1, \cdots, J$, i.e., $\boldsymbol{r}_{r}^{T}\left(s_{n}\right) \boldsymbol{v}_{\boldsymbol{k}}=0$ for $k=K+1, \cdots, J$ and $n=1,2, \cdots K$. Hence, $\boldsymbol{V}_{\boldsymbol{n}}^{\boldsymbol{T}} \boldsymbol{r}_{r}(s)=\mathbf{0}$ only when $s=$ $s_{1}, \cdots, s_{K}$. Therefore, $s_{k}$ can be obtained by finding $s$ that makes $\left\|\boldsymbol{V}_{\boldsymbol{n}}^{T} \boldsymbol{r}_{r}(s)\right\|=0$.

When noise exists, the orthogonality relations (17) no longer hold. In this case, we can search for signal vectors that are most closely orthogonal to the noise subspace. Hence, $s_{k}$ can be obtained by finding the peak of the following DMUSIC spectrum:

$$
P(s)=\frac{1}{\overline{\boldsymbol{r}}_{r}^{H}(s)\left(\sum_{k=K+1}^{J} \boldsymbol{v}_{k}^{*} \boldsymbol{v}_{k}^{T}\right) \overline{\boldsymbol{r}}_{r}(s)}
$$

where

$$
\overline{\boldsymbol{r}}_{r}=\frac{\boldsymbol{r}_{r}}{\left\|\boldsymbol{r}_{r}\right\|} .
$$

The algorithm is summarized in Table I. The algorithm discussed above is called the damped MUSIC (DMUSIC) algorithm. Since the mathematical model of NMR data has the damped sinusoidal form, it is true that it is nonstationary in a strict mathematical sense. This is easy to prove and it 
TABLE I

DAmped Music Algorithm

\begin{tabular}{ll|l}
\hline Step & 1 & Forming data matrix A using (9) \\
\hline Step & 2 & Finding $\mathbf{V}_{\mathbf{n}}$ by making SVD to $\mathbf{A}$ \\
\hline Step & 3 & Estimating $s_{k}$ by finding the peaks of (19) \\
\hline
\end{tabular}

follows directly from standard methods for computing the autocorrelation function for a random sequence and interested readers should refer to [30] and [31]. There are two crucial differences between the DMUSIC algorithm and the MUSIC algorithm. First of all, the DMUSIC algorithm is for parameter estimation of damped sinusoidal signals which are nonstationary, as opposed to MUSIC algorithm which works for stationary signals. Since the correlation matrix is not available for nonstationary signals, in DMUSIC algorithm the prediction matrix is used instead of correlation matrix. Second, the DMUSIC algorithm searches the $(\alpha, \omega)$ plane to estimate two parameters simultaneously.

\section{M-D DMUSIC ALGORITHM}

The DMUSIC algorithm for 1-D NMR signals developed in the Section III can be extended to the DMUSIC algorithm for multidimensional NMR signals (M-D DMUSIC).

To obtain a DMUSIC algorithm for the 2-D NMR signals modeled in (3) and (4), we first generate an $(N-J+1) \times J$ matrix, (21) shown at the bottom of the page, for $n=$ $1,2, \cdots, N-1$. Based on $\boldsymbol{A}(n)$, a $(N-J+1)^{2} \times J^{2}$ matrix is formed

$$
\boldsymbol{A}=\left(\begin{array}{cccc}
A(0) & \boldsymbol{A}(1) & \cdots & \boldsymbol{A}(J-1) \\
A(1) & A(2) & \cdots & A(J) \\
\vdots & \vdots & \vdots & \vdots \\
A(N-J-1) & A(N-J) & \cdots & A(N-1)
\end{array}\right)
$$

where $K \leq J \leq N-K$. It is important to notice that the rank of matrix $A$ is $K$.

Using a similar procedure to the derivation of 1-D DMUSIC algorithm, we can form a 2-D DMUSIC spectrum

$$
P(\boldsymbol{s})=\frac{1}{\overline{\boldsymbol{r}}_{r}^{H}(\boldsymbol{s})\left(\sum_{k=K+1}^{J^{2}} \boldsymbol{v}_{k}^{*} \boldsymbol{v}_{k}^{T}\right) \overline{\boldsymbol{r}}_{r}(\boldsymbol{s})}
$$

where $\boldsymbol{v}_{k}$ for $k=K+1, \cdots, J^{2}$ are the right singular vectors of $A$ corresponding to the $J^{2}-K$ smallest singular values and

$$
\begin{aligned}
\boldsymbol{r}_{r}\left(\boldsymbol{s}_{k}\right)= & \left(\begin{array}{llllll}
1 & e^{s_{k}^{(2)}} & \cdots & e^{(J-1) s_{k}^{(2)}} & \cdots & e^{(J-1) s_{k}^{(1)}} \\
& e^{(J-1) s_{k}^{(1)}+s_{k}^{(2)}} & \cdots & e^{(J-1) s_{k}^{(1)}+(J-1) s_{k}^{(2)}}
\end{array}\right)^{T}
\end{aligned}
$$

and $\overline{\boldsymbol{r}}_{r}=\boldsymbol{r}_{r} /\left\|\boldsymbol{r}_{r}\right\|$. The parameter $\boldsymbol{s}_{k}$ of 2-D NMR data can be estimated by finding $\boldsymbol{s}$ to maximize the above 2-D DMUSIC spectrum.

In general, for $L$-dimensional NMR signal, $(N-J+1)^{L} \times$ $J^{L}$ data matrix $\boldsymbol{A}$ is defined as

$$
\boldsymbol{A}=\left(\begin{array}{cccc}
A(0) & A(1) & \cdots & A(J-1) \\
A(1) & A(2) & \cdots & A(J) \\
\vdots & \vdots & \vdots & \vdots \\
A(N-J-1) & A(N-J) & \cdots & A(N-1)
\end{array}\right)
$$

where indexes $n_{1}, n_{2}, \cdots, n_{l}$ correspond to the number of given data points in each dimension, i.e., we are using an $n_{1} \times n_{2} \times \cdots \times n_{L}$ data matrix and (26), (27) shown at the bottom of the next page.

The right signal vector corresponding to the data matrix $A$ is defined as

$$
\boldsymbol{r}(\boldsymbol{s})=\left(\begin{array}{c}
\boldsymbol{r}_{L-1}(\boldsymbol{s}) \\
e^{\boldsymbol{s}_{k}^{(1)}} \boldsymbol{r}_{L-1}(\boldsymbol{s}) \\
\vdots \\
e^{(J-1) s_{k}^{(1)}} \boldsymbol{r}_{L-1}(\boldsymbol{s})
\end{array}\right)
$$

where

$$
\boldsymbol{r}_{l}(\boldsymbol{s})=\left(\begin{array}{c}
\boldsymbol{r}_{l}(\boldsymbol{s}) \\
e^{\boldsymbol{s}_{k}^{(L-l+1)}} \boldsymbol{r}_{l-1}(\boldsymbol{s}) \\
\vdots \\
e^{(J-1) s_{k}^{(L-i+1)}} \boldsymbol{r}_{l-1}(\boldsymbol{s})
\end{array}\right)
$$

for $l=2,3, \cdots, L-1$, and

$$
\boldsymbol{r}_{1}(\boldsymbol{s})=\left(\begin{array}{c}
1^{s^{(L)}} \\
\vdots \\
e^{(J-1) s_{k}^{(L)}}
\end{array}\right)
$$

For $J \geq K, J^{L} \times 1$ vectors $\boldsymbol{r}\left(\boldsymbol{s}_{k}\right)$ 's for $k=1,2, \cdots K$ are linearly independent vectors. Therefore, similar to 1-D DMUSIC algorithm, the frequency vector $s$ can be estimated by finding the peaks of M-D DMUSIC spectrum

$$
\begin{gathered}
P(\boldsymbol{s})=\frac{1}{\overline{\boldsymbol{r}}_{r}^{H}(\boldsymbol{s})\left(\sum_{L}^{J^{L}} k=K+1 \boldsymbol{v}_{k}^{*} \boldsymbol{v}_{k}^{T}\right) \overline{\boldsymbol{r}}_{r}(\boldsymbol{s})} \\
\text { and } \quad \overline{\boldsymbol{r}}_{r}=\frac{\boldsymbol{r}_{r}}{\left\|\boldsymbol{r}_{r}\right\|}
\end{gathered}
$$

where vectors $\boldsymbol{v}_{K+1}, \cdots, \boldsymbol{v}_{J^{L}}$ are $J^{L}-K$ right singular vectors of $\boldsymbol{A}$ corresponding to the $J^{L}-K$ smallest singular values.

$$
\boldsymbol{A}(n)=\left(\begin{array}{cccc}
y(n, 0) & y(n, 1) & \cdots & y(n, J-1) \\
y(n, 1) & y(n, 2) & \cdots & y(n, J) \\
\vdots & \vdots & \vdots & \vdots \\
y(n, N-J) & y(n, N-J+1) & \cdots & y(n, N-1)
\end{array}\right)
$$


TABLE II

2-D Peak-Searching Algorithm

\begin{tabular}{l|l}
\hline Step 1 & Find $\left(\widehat{\omega}_{k}^{(1)}, \widehat{\omega}_{k}^{(2)}\right)$ for $k=1,2, \cdots, K$ maximizing $P\left(0,0 ; \omega^{(1)}, \omega^{(2)}\right)$ \\
\hline Step 2 & Find $\left(\widehat{\alpha}_{1}^{(1)}, \widehat{\alpha}_{1}^{(2)}\right)$ maximizing $P\left(\alpha^{(1)}, \alpha^{(2)} ; \widehat{\omega}_{1}^{(1)}, \widehat{\omega}_{1}^{(2)}\right)$ \\
\hline Step 3 & Find $\left(\widehat{\hat{\omega}}_{1}^{(1)}, \widehat{\hat{\omega}}_{1}^{(2)}\right)$ around $\left(\widehat{\omega}_{1}^{(1)}, \widehat{\omega}_{1}^{(2)}\right)$ and maximizing $P\left(\widehat{\alpha}_{1}^{(1)}, \widehat{\alpha}_{1}^{(2)}, \omega^{(1)}, \omega^{(2)}\right)$ \\
\hline Step 4 & $\begin{array}{l}\text { Repeat Step } 2,3 \text { until the estimation of } s_{1}^{(1)}=-\alpha_{1}^{(1)}+\jmath \omega_{1}^{(1)} \\
(2)=-\alpha_{1}^{(2)}+\jmath \omega_{1}^{(2)} \text { attains certain precision }\end{array}$ \\
\hline Step 5 & Repeat Step 2, 3 and 4 for $k=2,3, \cdots, K$ \\
\hline
\end{tabular}

\section{LOW-COMPLEXITY 2-D PEAK-SEARCHING METHOD}

To estimate the parameters of L-D NMR signals, we have to find the peaks of L-D DMUSIC spectrum, which is a function of $2 L$ variables: $\alpha^{(1)}, \cdots, \alpha^{(L)}, \omega^{(1)}, \cdots, \omega^{(L)}$. To find the peak of the L-D DMUSIC spectrum, we have to calculate it in a fine lattice, which is a $2 \mathrm{~L}$-dimensional search.

Since the damping factors of NMR signals are normally very small (usually less than 0.3 ), the following simplified peak-searching algorithm can be used to reduce the computation of the 2-D DMUSIC spectrum. For convenience, we rewrite $P\left(s^{(1)}, s^{(2)}\right)$ as $P\left(\alpha^{(1)}, \alpha^{(2)} ; \omega^{(1)}, \omega^{(2)}\right)$. If the damping factors of signals are small, then the maxima of $P\left(\alpha^{(1)}, \alpha^{(2)} ; \omega^{(1)}, \omega^{(2)}\right)$ will be near the $\left(\omega^{(1)}, \omega^{(2)}\right)$ plane.

Because $P\left(\alpha^{(1)}, \alpha^{(2)} ; \omega^{(1)}, \omega^{(2)}\right)$ is convex around its maxima, $P\left(0,0 ; \omega^{(1)}, \omega^{(2)}\right)$ is also convex around the maxima. Hence, $P\left(0,0 ; \omega^{(1)}, \omega^{(2)}\right)$ has maximum points $\left(\hat{\omega}_{k}^{(1)}, \hat{\omega}_{k}^{(2)}\right)$ for $k=1,2, \cdots, K$, which are near the peaks of $P\left(\alpha^{(1)}, \alpha^{(2)} ; \omega^{(1)}, \omega^{(2)}\right)$. For each maximum $\left(\hat{\omega}_{k}^{(1)}, \hat{\omega}_{k}^{(2)}\right)$ of $P\left(0,0 ; \omega^{(1)}, \omega^{(2)}\right),\left(\hat{\alpha}_{k}^{(1)}, \hat{\alpha}_{k}^{(2)}\right)$ can be found to maximize $P\left(\alpha^{(1)}, \alpha^{(2)} ; \hat{\omega}_{k}^{(1)}, \hat{\omega}_{k}^{(2)}\right)$ since $P\left(\alpha^{(1)}, \alpha^{(2)} ; \hat{\omega}_{k}^{(1)}, \hat{\omega}_{k}^{(2)}\right)$ is convex. Then, we can find $\left(\hat{\omega}_{k}^{(1)}, \hat{\hat{\omega}}_{k}^{(2)}\right)$ around $\left(\hat{\omega}_{k}^{(1)}, \hat{\omega}_{k}^{(2)}\right)$ that maximizes $P\left(\hat{\alpha}_{k}^{(1)}, \hat{\alpha}_{k}^{(2)}, \omega^{(1)}, \omega^{(2)}\right)$. Repeating the above procedures, the peaks of $P\left(\alpha^{(1)}, \alpha^{(2)} ; \omega^{(1)}, \omega^{(2)}\right)$ can be searched. The above searching procedures, which are summarized in Table II, reduces the peak search of a fourvariable function to that of a two-variable function. Therefore, the computation is significantly reduced.

The parameter estimation of higher-dimensional NMR signals can be decomposed into parameter estimation of lowerdimensional NMR signals to reduce the computation. Taking three-dimensional (3-D) NMR signal $y\left(n_{1}, n_{2}, n_{3}\right)$ as an example, it is a 2-D NMR signal if $n_{3}$ is fixed. Hence, the frequency pairs corresponding to time index $n_{1}$ and $n_{2}$ can be estimated by the 2-D MUSIC algorithm. Similarly, the frequencies corresponding to $n_{3}$ can be estimated using the 1-D DMUSIC algorithm. The 3-D complex frequency vector can be found by searching the peaks of the 3-D DMUSIC spectrum near all the combinations of the frequency pairs corresponding to indices $n_{1}$ and $n_{2}$ and the frequencies corresponding to index $n_{3}$. In this way, parameter estimation of 3-D NMR signals is simplified into parameter estimation of 2-D NMR signals and 1-D NMR signals. In fact, the parameter estimation of higher-dimensional NMR signal can always be decomposed into the parameter estimations of corresponding 2-D NMR signals and 1-D NMR signal. It is also worth mentioning that the complexity of the $M$ $D$ DMUSIC algorithm is comparable to the other singularvalue decomposition (SVD)-based algorithms. Since in our algorithm high-resolution estimates can be achieved using fewer number of data points, the complexity of the SVD computation is less than that of existing methods whose performance relies on using large data matrices. But our algorithm also involves a 2L-D peak-searching process whose complexity is heavily reduced by our proposed peak-searching algorithm. Therefore, the burden of the peak-searching process is also significantly reduced in our algorithm.

\section{Simulation EXAMPLES}

Before presenting our estimation results for experimental data from NIH, we confirm the DMUSIC algorithms by three simulation examples in this section. In our simulation examples, the measurement noise $w(\cdot)$ is complex white Gaussian noise with variance $\sigma^{2}$ that is determined by peak

$$
A\left(n_{1}, \cdots, n_{l}\right)=\left(\begin{array}{ccc}
A\left(n_{1}, \cdots, n_{l}, 0\right) & \cdots & A\left(n_{1}, \cdots, n_{l}, J-1\right) \\
A\left(n_{1}, \cdots, n_{l}, 1\right) & \cdots & A\left(n_{1}, \cdots, n_{l}, J\right) \\
\vdots & \vdots & \vdots \\
A\left(n_{1}, \cdots, n_{l}, N-J-1\right) & \cdots & A\left(n_{1}, \cdots, n_{l}, N-1\right)
\end{array}\right)
$$

for $l=1,2, \cdots, L-2$

$$
\boldsymbol{A}\left(n_{1}, \cdots, n_{L-1}\right)=\left(\begin{array}{ccc}
y\left(n_{1}, \cdots, n_{L-1}, 0\right) & \cdots & y\left(n_{1}, \cdots, n_{L-1}, J-1\right) \\
y\left(n_{1}, \cdots, n_{L-1}, 1\right) & \cdots & y\left(n_{1}, \cdots, n_{L-1}, J\right) \\
\vdots & \vdots & \vdots \\
y\left(n_{1}, \cdots, n_{L-1}, N-J\right) & \cdots & y\left(n_{1}, \cdots, n_{L-1}, N-1\right)
\end{array}\right)
$$




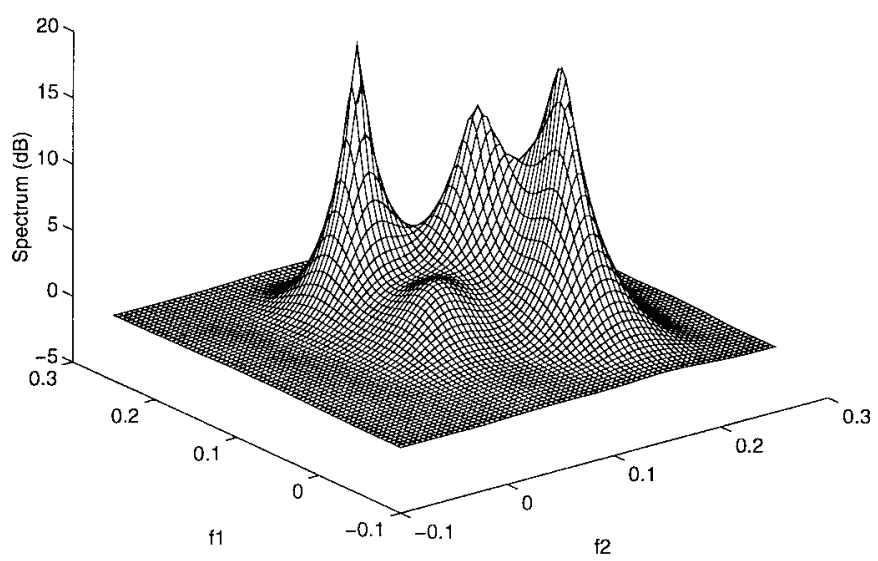

(a)

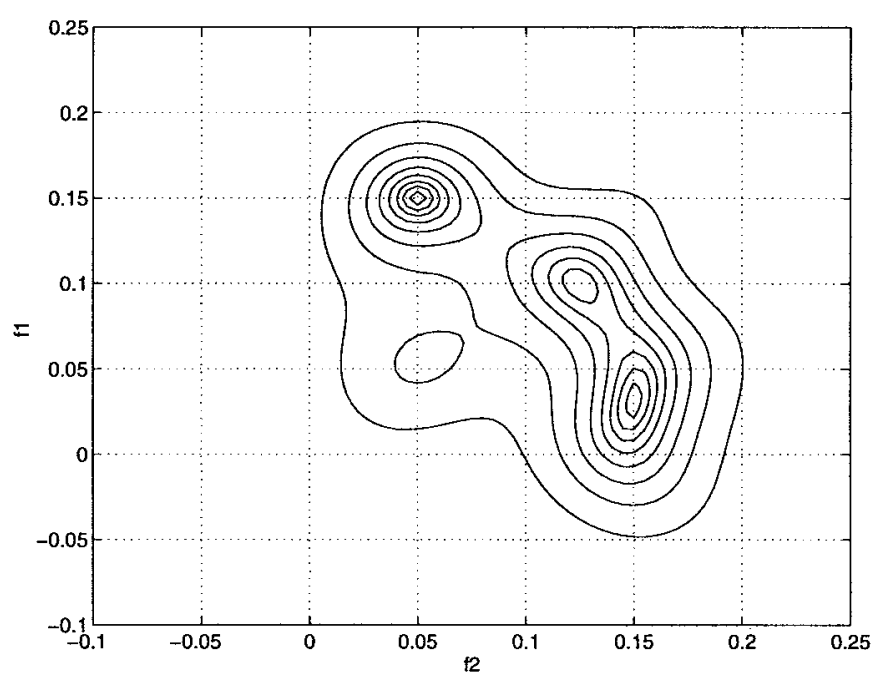

(b)

Fig. 2. The spectrum $P\left(0,0 ; \omega^{(1)}, \omega^{(2)}\right)$ and its contour for synthetic 2-D NMR signal using 2-D peak-searching method when SNR $=30 \mathrm{~dB}$.

SNR defined as

$$
\mathrm{SNR}=10 \log \frac{1}{2 \sigma^{2}} .
$$

Example 1: The synthetic 1-D NMR data is generated by

$$
y(n)=e^{s_{1} n}+e^{s_{2} n}+w(n)
$$

where $s_{1}=-0.2+j 2 \pi(0.42), s_{2}=-0.1+j 2 \pi(0.42+\Delta)$. The data length is $N=24$, therefore, we pick $J=12$.

For $\mathrm{SNR}=40 \mathrm{~dB}, \Delta=0.1$, the 1-D DMUSIC spectrum and its contour are shown in Fig. 1(a) and (b), respectively. From these figures, the damping factors and frequencies of the signal can be estimated simultaneously by finding the peak on the spectrum. But, if $\mathrm{SNR}=40 \mathrm{~dB}, \Delta=0$, i.e., two exponentially damped signals with the same frequency, the spectrum has just one peak, as indicated by Fig. 1(c) and (d). Hence, the damping factors of the signals cannot be estimated correctly under this condition. However, if SNR is increased to $60 \mathrm{~dB}$, both the damping factors and the frequencies of the signals can be estimated again as demonstrated by Fig. 1(e) and (f).

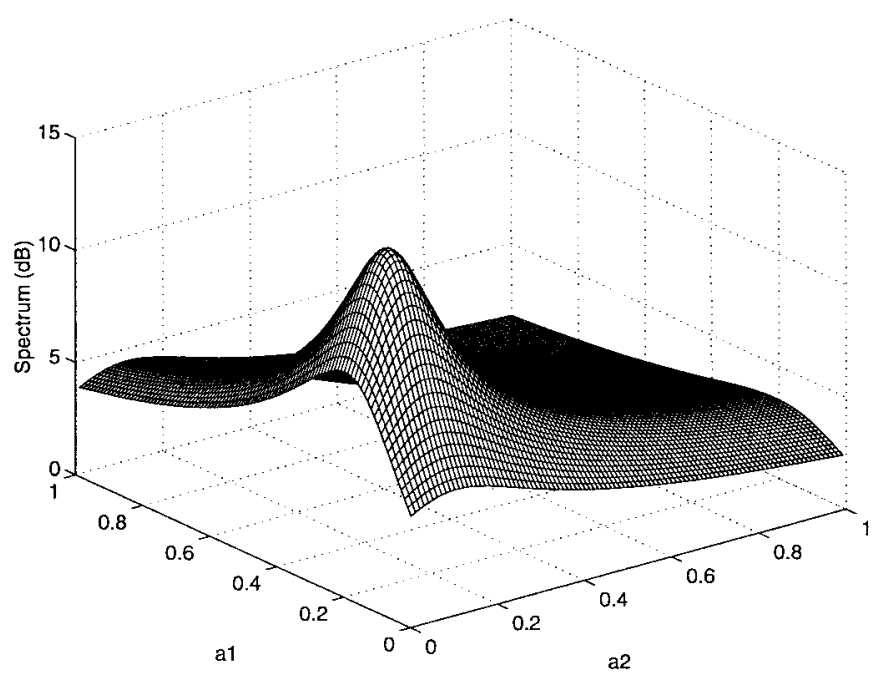

(a)

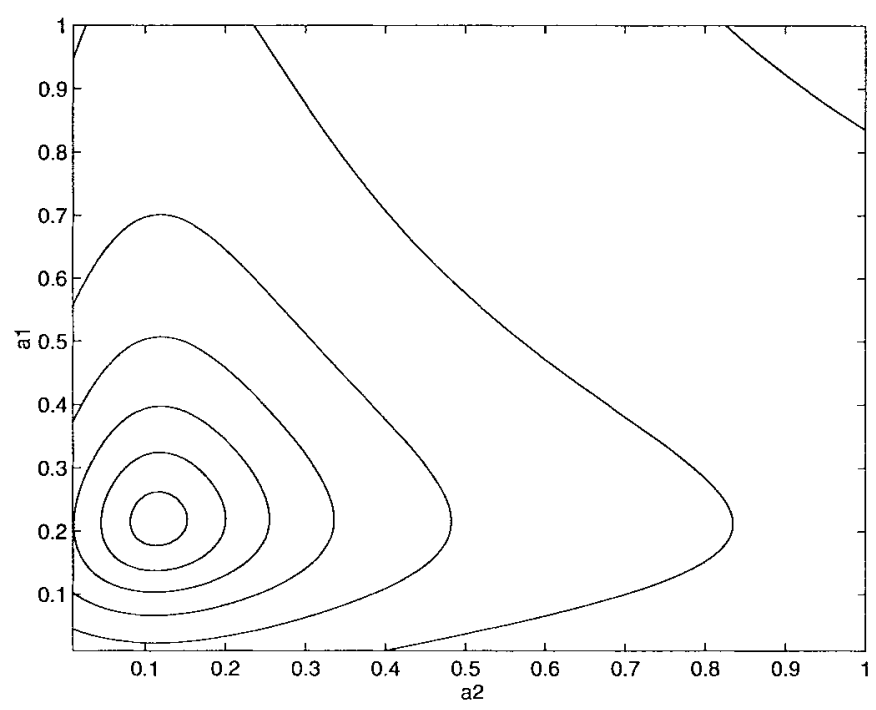

(b)

Fig. 3. The spectrum $P\left(\alpha^{(1)}, \alpha^{(2)} ; 0.20 \pi, 0.25 \pi\right)$ and its contour for synthetic 2-D NMR signal 2-D peak-searching method when SNR $=30 \mathrm{~dB}$.

Example 2: In this example, a set of synthetic 2-D NMR data is first generated using (3) and (4). The model order $K=5$ and the frequency pairs are shown in Table III. The data length is $N=24$ and $J$ is, therefore, chosen to be 12 to achieve best performance.

For $\mathrm{SNR}=40 \mathrm{~dB}$, the 2-D DMUSIC spectrum, $P(\boldsymbol{s})$, and its contour, using 2-D peak-searching algorithm are shown in Figs. 2 and 3. From the figures, we can see that $P(\boldsymbol{s})$ only has four peaks on $\omega_{1}-\omega_{2}$ plane with $\alpha^{(1)}=\alpha^{(2)}=0$, but five peaks are found by the 2-D peak-searching algorithm and the parameters can be estimated successfully as illustrated by Table III.

From the estimation results in Table III, our estimation algorithm cannot resolve the fourth and fifth frequency pairs when $\mathrm{SNR}=20 \mathrm{~dB}$, since they are to close.

Example 3: The 3-D synthetic NMR signal is generated by using (7) and (8). The model order is $K=3$. The frequency vectors are shown in Table IV. The synthetic NMR 


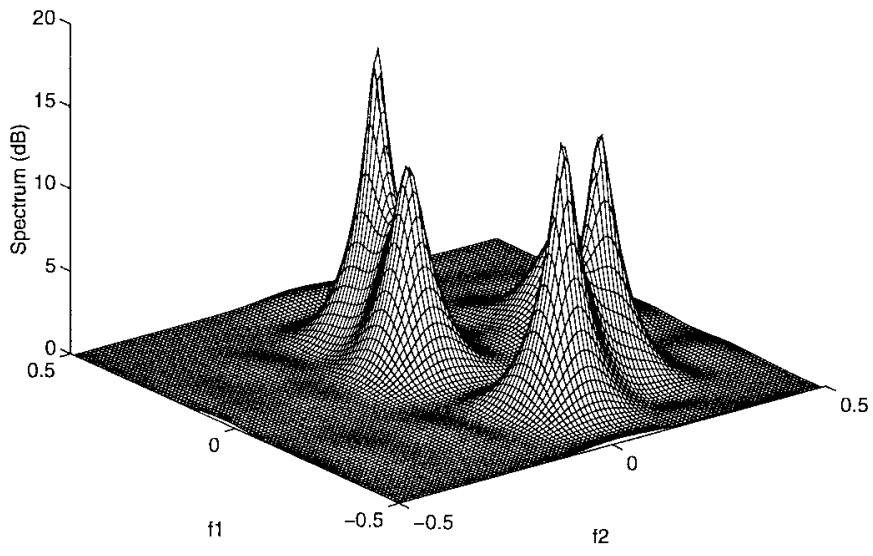

(a)

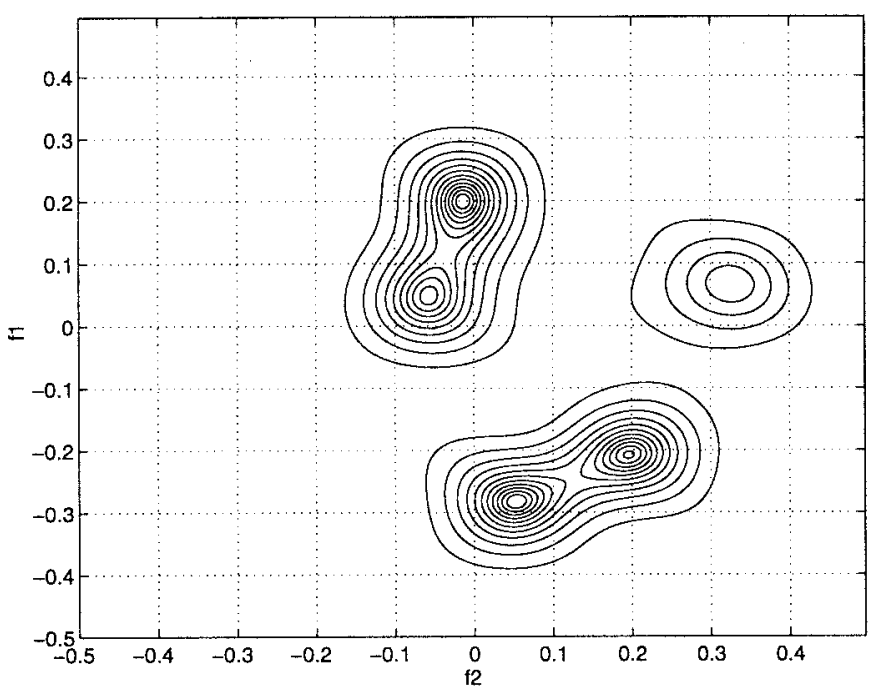

(b)

Fig. 4. The spectrum $P\left(0,0 ; \omega^{(1)}, \omega^{(2)}\right)$ and its contour for 2-D NMR signal.

TABLE III

Estimated Parameters of A Synthetic 2-D NMR Signal

\begin{tabular}{|c|c|c|c|c|c|}
\hline & $\mathrm{k}$ & $\alpha_{k}^{(1)}$ & $w_{k}^{(1)}$ & $\alpha^{(2)_{k}}$ & $\omega_{k}^{(2)}$ \\
\hline \multirow{5}{*}{$\begin{array}{l}\text { True } \\
\text { values }\end{array}$} & 1 & -0.20 & $0.10 \pi$ & -0.10 & $0.10 \pi$ \\
\hline & 2 & -0.00 & $0.30 \pi$ & -0.00 & $0.10 \pi$ \\
\hline & 3 & -0.05 & $0.20 \pi$ & -0.02 & $0.25 \pi$ \\
\hline & 4 & -0.02 & $0.05 \pi$ & -0.02 & $0.30 \pi$ \\
\hline & 5 & -0.10 & $0.06 \pi$ & -0.02 & $0.31 \pi$ \\
\hline \multirow{5}{*}{$\begin{array}{c}\text { Estimated } \\
\text { values } \\
\mathrm{SNR}=30 \mathrm{~dB}\end{array}$} & 1 & -0.21 & $0.10 \pi$ & "-0.11 & $0.10 \pi$ \\
\hline & 2 & -0.00 & $0.30 \pi$ & -0.00 & $0.10 \pi$ \\
\hline & 3 & -0.05 & $0.20 \pi$ & -0.02 & $0.25 \pi$ \\
\hline & 4 & -0.11 & $0.05 \pi$ & -0.04 & $0.31 \pi$ \\
\hline & 5 & -0.09 & $0.07 \pi$ & -0.01 & $0.31 \pi$ \\
\hline \multirow{5}{*}{$\begin{array}{c}\text { Estimated } \\
\text { values } \\
\mathrm{SNR}=20 \mathrm{~dB}\end{array}$} & $\overline{1}$ & $\overline{c-0.19}$ & $0.10 \pi$ & -0.10 & $0.10 \pi$ \\
\hline & 2 & -0.00 & $0.30 \pi$ & -0.00 & $0.10 \pi$ \\
\hline & 3 & -0.05 & $0.20 \pi$ & -0.02 & $0.25 \pi$ \\
\hline & 4 & -1.00 & $0.64 \pi$ & -0.00 & $0.31 \pi$ \\
\hline & 5 & -0.07 & $0.06 \pi$ & -0.01 & $0.31 \pi$ \\
\hline
\end{tabular}

signal is corrupted by measurement noise with $\mathrm{SNR}=15 \mathrm{~dB}$. First, we estimate the complex frequency pairs corresponding to the first two indexes using the simplified 2-D searching algorithm and the complex frequencies corresponding to the

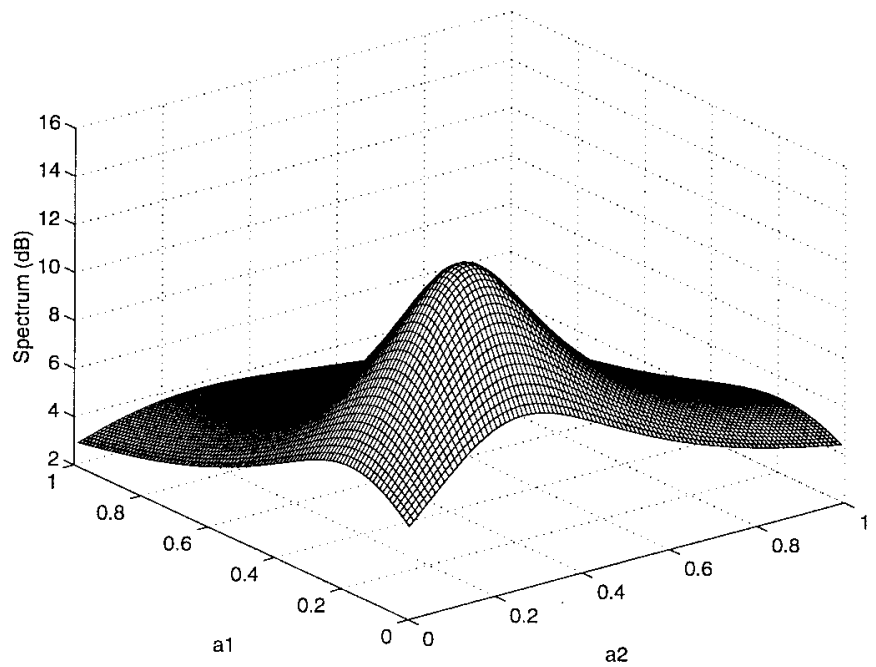

(a)

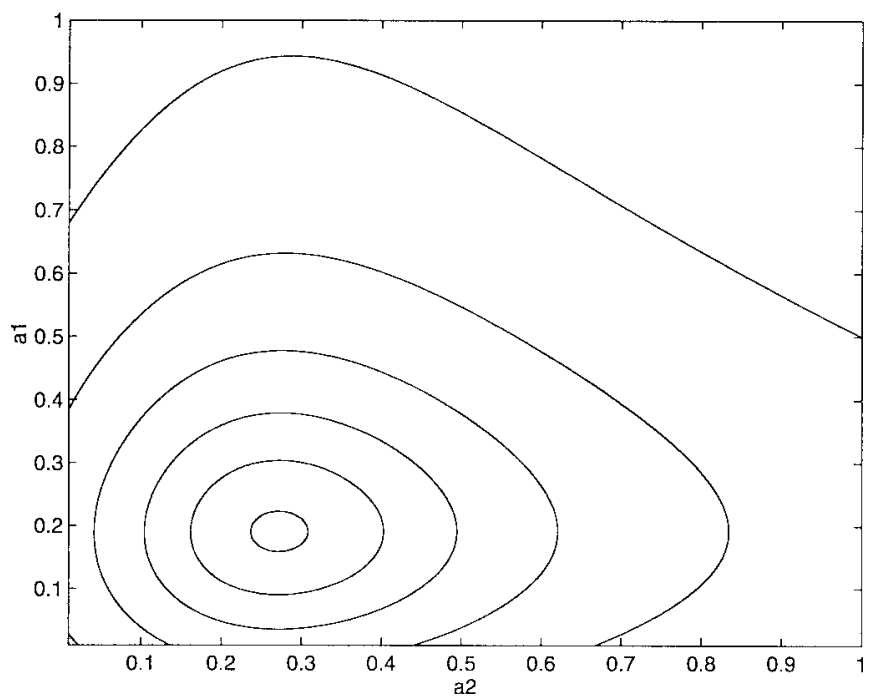

(b)

Fig. 5. The spectrum $P\left(\alpha^{(1)}, \alpha^{(2)} ; 0.10 \pi,-0.12 \pi\right)$ and its contour for 2-D NMR signal.

TABLE IV

Estimated Parameters of a Synthetic 3-D NMR Signal

\begin{tabular}{c||c||c|c|c|c|c|c}
\hline & $\mathrm{k}$ & $\alpha_{k}^{(1)}$ & $\omega_{k}^{(1)}$ & $\alpha^{(2)_{k}}$ & $\omega_{k}^{(2)}$ & $\alpha^{(3)_{k}}$ & $\omega_{k}^{(3)}$ \\
\hline \hline \multirow{2}{*}{$\begin{array}{c}\text { True } \\
\text { values }\end{array}$} & 1 & 0.040 & $-0.740 \pi$ & 0.140 & $-0.820 \pi$ & 0.100 & $-0.140 \pi$ \\
& 2 & 0.010 & $0.050 \pi$ & 0.190 & $-0.190 \pi$ & 0.170 & $0.310 \pi$ \\
& 3 & 0.140 & $0.290 \pi$ & 0.080 & $-0.720 \pi$ & 0.010 & $0.150 \pi$ \\
\hline \hline \multirow{2}{*}{$\begin{array}{c}\text { Estimated } \\
\text { values }\end{array}$} & 1 & 0.040 & $-0.740 \pi$ & 0.150 & $-0.815 \pi$ & 0.100 & $-0.140 \pi$ \\
\cline { 2 - 8 } SNR $=15 \mathrm{~dB}$ & 2 & 0.010 & $0.050 \pi$ & 0.190 & $-0.190 \pi$ & 0.180 & $0.310 \pi$ \\
\cline { 2 - 8 } & 3 & 0.140 & $0.295 \pi$ & 0.080 & $-0.720 \pi$ & 0.020 & $0.145 \pi$ \\
\hline
\end{tabular}

third index. Then, we find the 3-D frequency vectors by searching the maximum points at the all possible combinations of the complex frequency pairs and the complex frequencies. The estimated frequency vectors are shown in Table IV.

\section{ESTIMATION RESULTS ON EXPERIMENTAL DATA}

The 2-D MUSIC parameter estimation algorithm is used in experimental NMR data. The measured $24 \times 242$-D NMR 


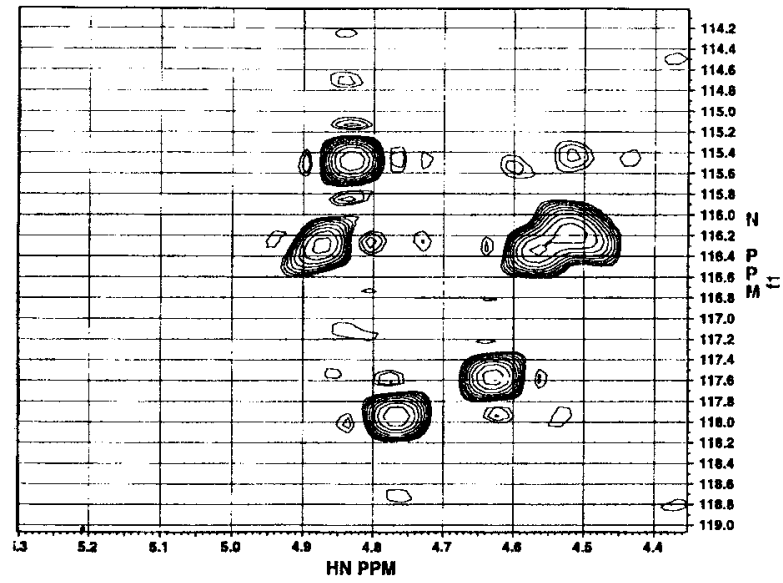

(a)

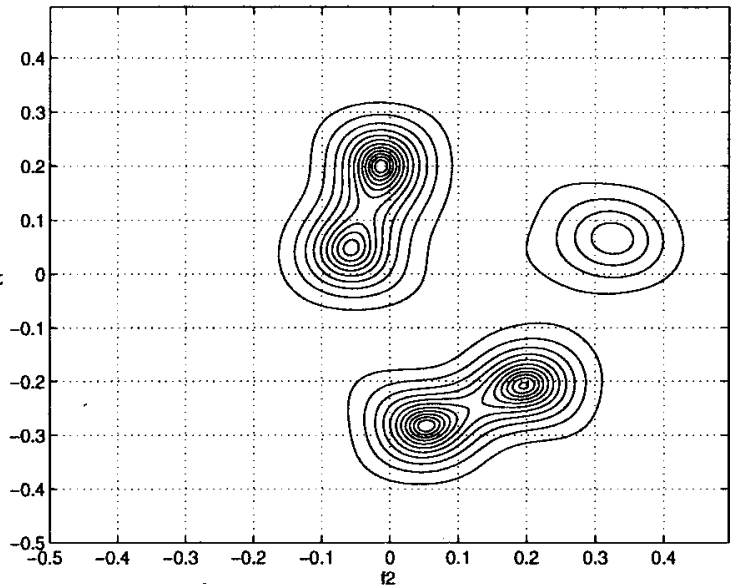

(b)

Fig. 6. Contour plot of $P\left(0,0 ; \omega^{(1)}, \omega^{(2)}\right)$ for 2-D NMR signal using (a) NmrPipe and (b) our 2-D DMUSIC algorithm.

TABLE V

Estimated Parameters of a Set OF EXPerimental 2-D NMR Data

\begin{tabular}{c||c|c|c|c}
\hline $\mathrm{k}$ & $\alpha_{k}^{(1)}$ & $\omega_{k}^{(1)}$ & $\alpha^{(2)_{k}}$ & $\omega_{k}^{(2)}$ \\
\hline \hline 1 & 0.06 & $0.04 \pi$ & 0.06 & $-0.02 \pi$ \\
\hline 2 & 0.07 & $-0.56 \pi$ & 0.08 & $0.10 \pi$ \\
\hline 3 & 0.07 & $-0.42 \pi$ & 0.09 & $0.40 \pi$ \\
\hline 4 & 0.13 & $0.10 \pi$ & 0.09 & $-0.12 \pi$ \\
\hline 5 & 0.21 & $0.12 \pi$ & 0.29 & $0.64 \pi$ \\
\hline
\end{tabular}

data was obtained from NIH's multidimensional NMR spectroscopy. The spectrum $P\left(0,0 ; \omega^{(1)}, \omega^{(2)}\right)$, and its contour are shown in Fig. 4(a) and (b). From Fig. 4, $P\left(0,0 ; \omega^{(1)}, \omega^{(2)}\right)$ has five peaks. The 2-D spectrum $P\left(\alpha^{(1)}, \alpha^{(2)} ; 0.10 \pi,-0.12 \pi\right)$ and its contour are shown in Fig. 5(a) and (b). By repeating Steps 2 and 3 in Table II just twice, we can successfully estimate the frequencies and the damping factors of the NMR data, which are listed in Table V.

Fig. 6 compares our algorithm with the present algorithm in the NmrPipe, which is a standard NMR processing tool in NIH used by the NMR researchers over the world. Fig. 6(a) and (b) illustrate the estimation results for the same set of experimental NMR data, respectively, by the present algorithm in the NmrPipe tool and the 2-D MUSIC algorithm. Compared with Fig. 6(b), Fig. 6(a) has many spurious signals, while our 2-D MUSIC algorithm can estimate signal parameters effectively without introducing any spurious signal. Hence, our method has been included in the NmrPipe tool as an effective algorithm for NMR data processing.

\section{CONCLUSION}

In this paper, we have proposed a high-resolution technique for multidimensional NMR spectroscopy, which we call the M-D MUSIC algorithm. Since the M-D DMUSIC algorithm makes full use of the rank-deficiency and Hankel properties of the prediction matrix composed of NMR data, it can estimate the NMR signal parameters under low SNR by using very few data points. To reduce the computational complexity, we have also introduced a peak-searching method to locate the peaks of the M-D DMUSIC spectrum.
The performance of our algorithms have been tested by extensive simulation examples and experimental data. The testing results show that the M-D DMUSIC algorithm can resolve closely-spaced frequencies and damping factors, which is one of the most effective algorithms for NMR data. Hence, it has been included in the NmrPipe tool in NIH and will be used by the NMR researchers over the world.

\section{ACKNOWLEDGMENT}

The authors would like to thank Dr. A. Bax from National Institute of Health for providing the experimental 2-D NMR data.

\section{REFERENCES}

[1] R. R. Enrst, Advances in Magnetic Resonance, Vol. 2. New York: Academic, 1966.

[2] J. C. Lindon and A. G. Ferrige, "Digitization and data processing in Fourier transform NMR," Progr. NMR Spectrosc., vol. 14, pp. 27-66, 1980.

[3] S. M. Kay and S. L. Marple, Jr., "Spectrum analysis-A modern perspective," Proc. IEEE, vol. 69, pp. 1380-1418, 1981.

[4] R. Kumaresan and R. W. Tufts, "Estimating the parameters of exponentially damped sinusoids and pole-zero modeling in noise," IEEE Trans. Acoust., Speech, Signal Processing, vol. ASSP-30, pp. 833-840, Dec. 1982.

[5] R. W. Tufts and R. Kumaresan, "Estimation of frequencies of multiple sinusoids: Making linear prediction perform like maximum likelihood," Proc. IEEE, vol. 70, pp. 975-989, 1982.

[6] Y. Bresler and A. Macovski, "Exact maximum likelihood parameter estimation of superimposed exponential signals in noise," IEEE Trans. Acoust., Speech, Signal Processing, vol. ASSP-34, pp. 1081-1089, Oct. 1986.

[7] M. A. Rahman and K. B. Yu, "Total least squares approach for frequency estimation using linear prediction," IEEE Trans. Acoust., Speech, Signal Processing, vol. ASSP-35, pp. 1440-1454, Oct. 1987.

[8] D. S. Stephenson, "Linear prediction and maximum entropy methods in NMR spectroscopy," Progr. NMR Spectrosc., vol. 20, pp. 515-626, 1988.

[9] S. A. Meyer, D. L. Orth, M. A. Morgenstern, and D. W. Noid, "MUSIC analysis applied to NMR," J. Magn. Reson. vol. 86, pp. 406-409, 1990.

[10] L. Mitschang, C. Cieslar, T. A. Holak, and H. Oschkinat, "Application of the Karhunen-Loeve transformation to the suppression of undesired resonances in the three-dimensional NMR," J. Magn. Reson. vol. 92, pp. 208-217, 1991.

[11] W. Dreher, P. Bornert, and D. Leibfritz, "Signal dependent preprocessing of NMR data using the constrained least square method," J. Magn. Reson., vol. 97, pp. 376-381, 1992. 
[12] V. U. Reddy and L. B. Biradar, "SVD-based information theoretic criteria for detection of the number of damped/undamped sinusoids and their performance analysis," IEEE Trans. Signal Processing, vol. 41, pp. 2872-2881, Sept. 1993.

[13] H. Gesmar and J. J. Led, "Spectral estimation of two-dimensional NMR signal-apply linear prediction to both dimensions," J. Magn. Reson. vol. 76, pp. 575-586, 1988

[14] C. F. Tirendi and J. F. Martin, "Fast linear prediction processing in two-dimensional NMR spectroscopy," J. Magn. Reson., vol. 81, pp. 577-585, 1989

[15] M. M. Barbieri and P. Barone, "A two-dimensional Prony's method for spectral estimation," IEEE Trans. Signal Processing, vol. 40, pp. 2747-2756, 1992

[16] Y. Hua, "Estimating two-dimensional frequencies by matrix enhancement and matrix pencil," IEEE Trans. Signal Processing, vol. 40, pp. 2267-2280, 1992

[17] J. J. Sacchini, W. M. Steedly, and R. L. Moses, "Two-dimensional Prony modeling and parameter estimation," IEEE Trans. Signal Processing, vol. 41, pp. 3127-3137, 1993.

[18] S. Van Huffel, H. Chen, C. Decanniere, and P. Van Hecke, "Algorithm for time-domain NMR data fitting based on total least squares," J. Magn. Reson., vol. 110, pp. 228-237, 1994.

[19] R. Schmidt, "Multiple emitter location and signal parameter estimation," IEEE Trans. Antennas Propagat., vol. AP-34, pp. 276-280, 1986.

[20] S. Haykin, Adaptive Filter Theory. Englewood Cliffs, NJ: PrenticeHall, 1991.

[21] H. Barkhuijsen, R. De Beer, and D. Van Ormondt, "Improved algorithm for noniterative time-domain model fitting to exponentially damped magnetic resonance signals," J. Magn. Reson., vol. 73, p. 553, 1987.

[22] W. Pijnappel, A. Van Den Boogaart, R. De Beer, and D. Van Ormondt, "SVD-based quantification of magnetic resonance signals," J. Magn. Reson., vol. 97, p. 122, 1992.

[23] A. Bax, "A simple description of two-dimensional NMR spectroscopy," Bull. Magn. Reson., vol. 7, pp. 167-183, 1985.

[24] J. Nuzillard and R. Freeman, "Oversampling in two-dimensional NMR," J. Magn. Reson., vol. 110, pp. 252-256, 1994.

[25] M. A. Delsuc, "Spectral representation of 2D NMR spectra by hypercomplex numbers," J. Magn. Reson., vol 77, pp. 119-124, 1988.

[26] Y. Li, K. J. R. Liu, and J. Razavilar, "A parameter estimation scheme for damped sinusoidal signals based on low-rank Hankel approximation," IEEE Trans. Signal Processing, vol. 45, pp. 481-486, Feb. 1997.

[27] S. Y. Kung, K. S. Arun, and D. V. Bhaskar Rao, "State-space and singular-value decomposition-based approximation methods for the harmonic retrieval problem," J. Opt. Soc. Amer., vol. 73, pp. 1799-1811, 1983.

[28] G. W. Stewart, "Perturbation theory for the singular value decomposition," in SVD and Signal Processing, II. Amsterdam, the Netherlands: : Elsevier Science, 1991, pp. 99-109.

[29] G. H. Golub and C. F. Van Loan, Matrix Computation, 2nd ed. Baltimore, MD: Johns Hopkins Press, 1989.

[30] A. Leon-Garcia, Probability and Random Processes for Electrical Engineering. Reading, MA: Addison-Wesley, 1994.

[31] A. V. Balakrishnan, Introduction to Random Processes in Engineering. New York: Wiley, 1995.

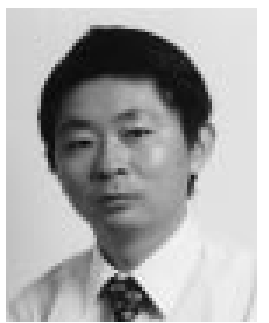

Ye (Geoffrey) Li was born in Jiangsu, China. He received the B.Eng. and M.Eng. degrees in 1983 and 1986, respectively, from the Department of Wireless Engineering, Nanjing Institute of Technology, Nanjing, China. He received the Ph.D. degree in 1994 from the Department of Electrical Engineering, Auburn University, Auburn, AL.

From March 1986 to May 1991, he was a Teaching Assistant and then a Lecturer with the National Mobile Communication Laboratory, Southeast University, China. From September 1991 to September 1994, he was a Research and Teaching Assistant with the Department of Electrical Engineering, Auburn University. From September 1994 to May 1996. He was a Post-Doctoral Research Associate with the Department of Electrical Engineering and Institute for Systems Research, University of Maryland at College Park. Since May 1996, he has been in Wireless Systems Research Department, AT\&T Labs-Research, Redbank, NJ. His general research interests include digital communications, wireless mobile systems, and statistical signal processing and its applications.

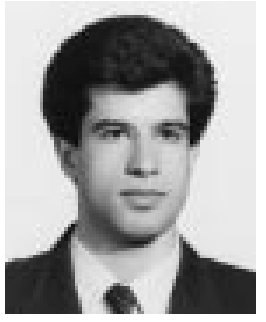

Javad Razavilar received the B.Sc. degree in electrical engineering from Sharif University of Technology, Tehran, Iran, in 1989. Since 1993, he has pursued graduate study at the University of Maryland at College Park, where he received the M.Sc. degree in electrical engineering in 1995 . He is currently completing the Ph.D. degree in electrical engineering at the same university while holding a Graduate Research Assistantship from Institute for Systems Research.

Mr. Razavilar is a member of IEEE Signal Processing Society. His research interests are in the fields of statistical signal processing, digital communications, and wireless networks.

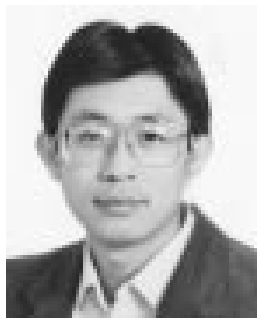

K. J. Ray Liu (S'86-M'86-SM'93) received the B.S. degree from the National Taiwan University in 1983 and the Ph.D. degree from the University of California, Los Angeles, in 1990, both in electrical engineering.

Since 1990 he has been with Electrical Engineering Department and Institute for Systems Research of University of Maryland at College Park, where he is an Associate Professor. During his sabbatical leave in 1996-97, he was Visiting Associate Professor at Stanford University, Stanford, CA. His research interests span all aspects of signal processing with application to image and video, wireless communications, networking, and medical and biomedical technology.

Dr. Liu has received numerous awards, including: the 1994 National Science Foundation Young Investigator Award, the IEEE Signal Processing Society's 1993 Senior Award (Best Paper Award), the George Corcoran Award in 1994 for outstanding contributions to electrical engineering education and the 1995-96 Outstanding Systems Engineering Faculty Award in recognition of outstanding contributions in interdisciplinary research, both from the University of Maryland. He is an Associate Editor of IEEE Transactions ON Signal PROCESSING, a Guest Editor of special issues on Multimedia Signal Processing and Technology of ProceEDINGS OF THE IEEE, a Guest Editor of special issue on Signal Processing for Wireless Communications of IEEE TRANSACTIONS ON Very Large Scale Integration (VLSI) Systems, a member of the Design and Implementation of Signal Processing Systems Technical Committee, and a founding member of Multimedia Signal Processing Technical Committee of IEEE Signal Processing Society. 